\title{
Attenuation of scopolamine-induced deficits in pigeons' attention to delayed matching-to-sample cues by D-cycloserine
}

\author{
ANGELA C. RUSKE and K. GEOFFREY WHITE \\ University of Otago, Dunedin, New Zealand
}

\begin{abstract}
The ability of D-cycloserine to improve delayed matching accuracy and to reverse scopolamineinduced deficits in delayed matching-to-sample performance in pigeons was investigated. Dose levels were $0,2,4$ and $6 \mathrm{mg} / \mathrm{kg}$ of D-cycloserine and $0.025 \mathrm{mg} / \mathrm{kg}$ of scopolamine. Drugs were administered via the intraperitoneal cavity at a constant volume of $1 \mathrm{ml} / \mathrm{kg}$. Administration of scopolamine impaired matching accuracy across all retention intervals. D-Cycloserine attenuated the scopolamine-induced deficit. Higher order analyses revealed that initial discriminability, but not rate of forgetting, was affected by scopolamine and D-cycloserine administration. These results indicate that D-cycloserine is capable of reversing scopolamine-induced deficits in matching accuracy and suggest that a synergistic relationship may exist between the cholinergic and glutamatergic systems.
\end{abstract}

The NMDA ( $N$-methyl-D-aspartate) receptor, an excitatory amino acid receptor subtype, has been shown to be important in learning and memory (Collingridge \& Bliss, 1987; Monahan, Handelmann, Hood, \& Cordi, 1989). Numerous studies show that drugs that affect NMDA receptor action also disrupt memory and learning (Abraham, 1988; Abraham \& Mason, 1988; Davis, Butcher, \& Morris, 1992; Morris, 1989; Wong et al., 1986). A mechanism associated with NMDA receptor action is long-term potentiation (LTP), a phenomenon resulting in a lasting increase in synaptic efficacy as the result of brief high-frequency activity of input fibers (Abraham, 1988). This activity-dependent change in synaptic efficacy has been suggested to underlie the physiological basis of memory storage in the brain (Collingridge \& Bliss, 1987). Drugs that act as agonists at the NMDA receptor complex have been shown to facilitate cognition in a variety of behavioral procedures (Baxter et al., 1994; Flood, Baker, \& Davis, 1990; Handelmann, Nevins, Mueller, Arnolde, \& Cordi, 1989).

Over the years there has been increasing evidence to suggest that a synergistic interaction may exist between the cholinergic and glutamatergic systems, at least in visual recognition memory (Aigner, 1995). For example, Segal and Auerbach (1997) reported two new actions of acetylcholine (ACh). First, a fast-onset, short-lived increase in cellular responses to action at the NMDA receptor, and second, a slow-onset, long-lasting increase in reactivity to afferent stimulation. They described this second mechanism as having similar properties to tetani-

We thank Barry Dingwall and his team for technical assistance. Correspondence should be addressed to A. C. Ruske or K. G. White, Department of Psychology, University of Otago, Dunedin, New Zealand (e-mail: kgwhite@otago.ac.nz). cally induced LTP, and thus referred to it as muscarinic long-term potentiation (LTPm). Further, muscarinic agonists have been shown to facilitate LTP induction in the rat dentate gyrus (Burgard \& Sarvey, 1990), physostigmine (an acetylcholinesterase inhibitor) has been found to cause potentiation of population spikes resembling LTP (Ito, Miura, \& Kadokawa, 1988; Levkovitz \& Segal, 1994), and anticholinergic drugs have suppressed the ability of area CA1 to express LTP (Blitzer, Gil, \& Landau, 1990).

To date there have been a number of studies that have examined the effects of glutamatergic agonists on cognitive performance under conditions where the cholinergic system has been disrupted. In particular, D-cycloserine (DCS), a partial agonist at the glycine modulatory site of the NMDA receptor complex, has been shown to reverse scopolamine-induced deficits in a variety of species. For example, Matsuoka and Aigner (1996) found that DCS reversed the detrimental effects of scopolamine, a centrally acting cholinergic antagonist, in a delayed spatial matching task using monkeys. Ohno and Watanabe (1996) found DCS effective at reversing scopolamine-induced impairment in a three-panel runway setup task using rats. DCS has also been shown to reverse the deficits associated with scopolamine administration using humans (Jones, Wesnes, \& Kirby, 1993).

Scopolamine is a useful model for studying the effects of cognitive enhancers (Iversen, 1997). It has been shown to disrupt memory performance in pigeons (Ruske, Fisher, \& White, 1997), rats (Dunnett, 1985; Kirk, White, \& McNaughton, 1988; Miyamoto et al., 1996), monkeys (Bartus, 1978; Glick \& Jarvik, 1970; Penetar \& McDonough, 1983), and humans (Safer \& Allen, 1971). The aim of the present study was to investigate the ability of DCS to enhance pigeons' performance in a delayed matchingto-sample procedure and to reverse the deficits associated 
with scopolamine administration, relative to a vehicle control condition.

\section{METHOD}

\section{Subjects}

Three adult homing pigeons weighing between 530 and $590 \mathrm{~g}$, with previous experience in delayed matching-to-sample and prior history of drug administration, were used as subjects. The pigeons were maintained at $85 \%$ of their free-feeding weight by supplementary feeding of mixed grain. Water and grit were available at all times in their home cages. Each bird was run 7 days a week unless its weight was $12 \mathrm{~g}$ above or below the prescribed range.

\section{Apparatus}

A light- and sound-attenuating experimental chamber $(33 \mathrm{~cm}$ wide, $33 \mathrm{~cm}$ deep, and $34 \mathrm{~cm}$ high) contained an interface panel on one wall. The interior of the chamber was painted matt black, and a ventilation fan was situated in the right rear of the chamber opposite the interface panel. A Plexiglas response key, $2.2 \mathrm{~cm}$ in diameter, was mounted $22 \mathrm{~cm}$ above the grid floor in the center of the panel. A force of $0.15 \mathrm{~N}$ was required to activate the microswitch behind the center key. Two side keys, both $2.9 \mathrm{~cm}$ in diameter, were located $10 \mathrm{~cm}$ on either side of the center key, $22 \mathrm{~cm}$ above the grid floor. A force of approximately $0.2 \mathrm{~N}$ was required to operate the reed relay behind the side keys. The two side keys could be illuminated green or red by the lamps mounted behind them. Kodak wratten filters with peak wavelengths of 555 and $606 \mathrm{~nm}$ produced the red and green stimuli presented on the center key.

Wheat could be obtained from a mechanical (Campden Instruments) hopper via an aperture $(5 \mathrm{~cm}$ wide $\times 6 \mathrm{~cm}$ high) located directly below the center key, $5 \mathrm{~cm}$ from the grid floor. White-light illumination of the aperture and the offset of the key stimulus accompanied wheat presentation. Experimental events were controlled and recorded by a 486 PC computer and associated interfacing located in an adjoining room. The computer ran time-shared med-pc software.

\section{Procedure}

Daily experimental sessions consisted of 80 trials or $50 \mathrm{~min}$, whichever came first. Each trial began with red or green illumination of the center key (sample stimulus). After five pecks on the center key, the chamber was darkened and a retention interval of $0.2,1.5,3,6$, or $12 \mathrm{sec}$ was initiated. All five retention intervals were scheduled to occur equally often and in random order within each session. All responses during the retention interval were ineffective. Following the retention interval the two side keys were illuminated red and green. A correct matching response occurred if the bird pecked the hue presented during the sample phase. Correct responses were reinforced with 2.5 -sec access to wheat. Incorrect responses resulted in a 2.5 -sec blackout period. Trials were separated by a 15 -sec intertrial interval where the chamber was darkened and all responses were ineffective. The red and green choice stimuli were presented equally often on left and right keys for each retention interval. Preliminary baseline training in this procedure continued for 30 sessions before the first drug administration sessions were arranged.

\section{Drug Administration}

DCS and scopolamine were diluted to their required concentrations with double distilled water (DDW) and physiological saline, respectively. The concentrations used were 0 (vehicle) 2,4 , and $6 \mathrm{mg} / \mathrm{ml}$ of DCS and $0.025 \mathrm{mg} / \mathrm{ml}$ of scopolamine. The administration of the different dose levels of DCS and scopolamine plus DCS occurred in ascending and then descending order, with each condition starting and finishing with a vehicle control. The order of drug administration was the same for each bird. Drug sessions oc- curred on Mondays, Wednesdays, and Fridays, with baseline training occurring on the 4 nondrug days. DCS alone was tested before scopolamine plus DCS, and was conducted over 17 sessions (including 8 drug and 9 baseline sessions). Scopolamine-plus-DCS testing began 31 sessions after the completion of DCS alone and was conducted over 22 sessions (including 10 drug and 9 baseline sessions). In total, 70 sessions were conducted throughout the course of the experiment.

\section{Data Analysis}

Total correct and error matching responses were collapsed over trial type (red vs. green samples) at each retention interval for the different experimental conditions. Proportions of correct responses were calculated by dividing the total number of correct matching responses (red plus green samples) by the total number of responses (correct plus error) at each retention interval. Group data were obtained by averaging individual proportion correct scores.

Because the proportion correct measure is susceptible to response bias and is bounded at 1.0, we also report estimates of discriminability derived from the choice theory of signal detection (White, 1991). The discriminability measure does not have an upper bound and is therefore a more sensitive measure at high levels of accuracy. Discriminability measures were calculated for individual birds and averaged across birds to obtain group means at each retention interval. In order to account for zeros appearing in some cells, which results in indeterminate measures, 0.5 was added to each total of every cell (Hautus, 1995). Estimates of discriminability at each retention interval $(\log d)$ were derived from correct (c) and error (e) responses following each sample $(1,2)$ according to Equation 1 (White, 1985).

$$
\log d=0.5 \times \log (\mathrm{cl} / \mathrm{el} \times \mathrm{c} 2 / \mathrm{e} 2) .
$$

Higher order measures of performance were achieved by fitting a negative exponential function to the individual and group mean discriminability measures $y$ for the different retention intervals $t$ (White, 1985; White \& Harper, 1996). This function is given by Equation 2:

$$
y=a \times \exp (-b \times \sqrt{t}) .
$$

The advantage of fitting such a function to the data is that it allows an assessment of the rate of forgetting independently of overall level of performance, or initial discriminability (discriminability at time $t=0$ ). These independent aspects of remembering are estimated by the parameter $b$ and $a$, respectively, of the fitted functions (White, 1985). The utility of this higher order analysis is that it separates encoding or attentional aspects of performance, measured by initial discriminability, from retrieval or memorial aspects, measured by rate of forgetting. These different aspects of delayed matching-to-sample performance are often sensitive to the effects of different drugs (White, Ruske, \& Colombo, 1996). For example, the attentional component as measured by the initial discriminability parameter $a$ is influenced by scopolamine (Kirk et al., 1988), whereas rate of forgetting, as measured by the parameter $b$, is influenced by chlorpromazine and phenobarbital (Watson \& Blampied, 1989; White, Harper, \& Watson, 1994).

Proportion correct responses, discriminability measures $(\log d)$, and derived initial discriminability and rate of forgetting parameters were submitted to a repeated measures analysis of variance (ANOVA) with dose and retention interval as the independent variables. A criterion of $p<.05$ was used for significance.

\section{RESULTS}

Figure 1 shows group average proportion correct responses as a function of retention interval for each dose level of DCS alone and each dose level of DCS combined 


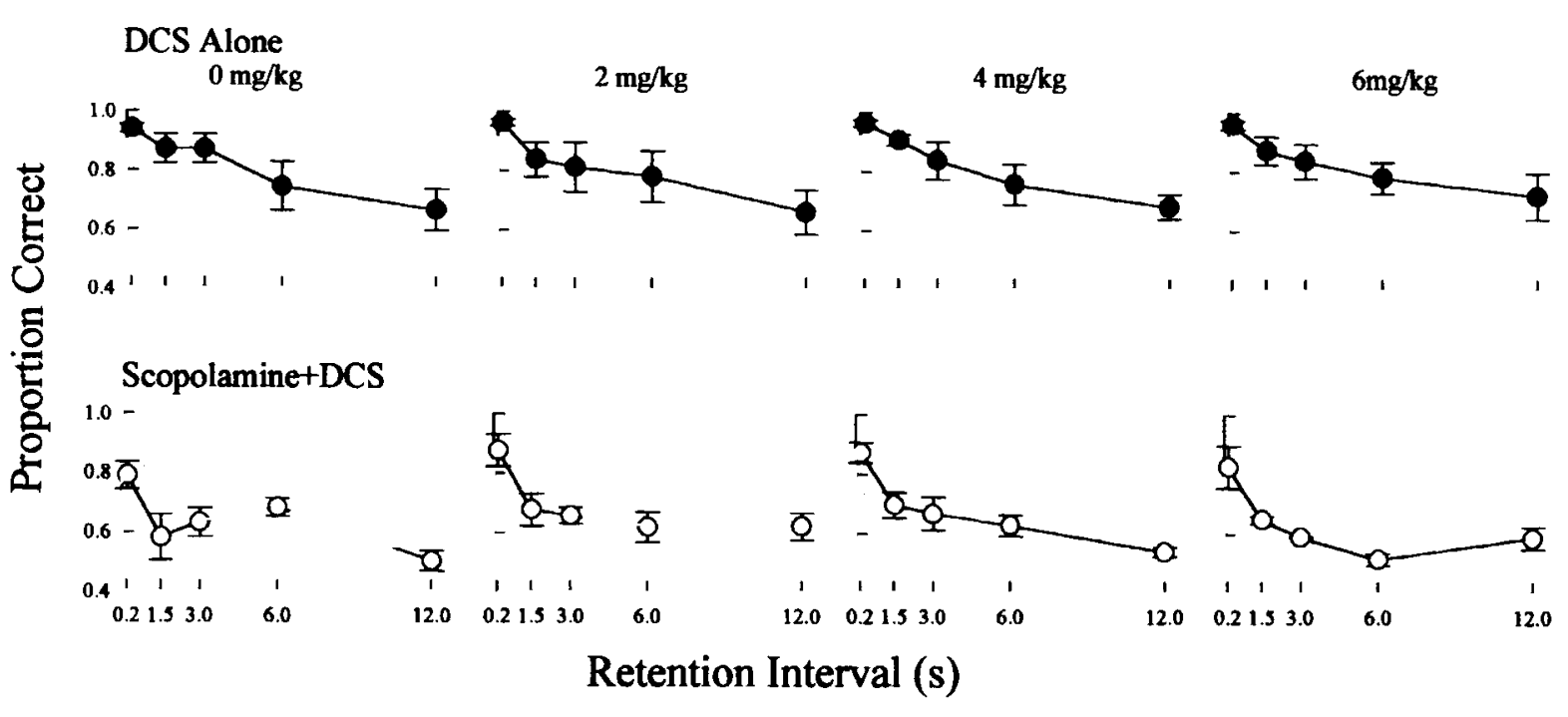

Figure 1. Group average proportion correct responses as a function of retention interval for each dose level of b-cycloserine (DCS) -alone and scopolamine-plus-DCS conditions. Vertical lines refer to standard errors of the mean.

with scopolamine. The results of the vehicle control for the scopolamine condition are not shown in Figure 1. Separate repeated measures ANOVAs were conducted for the DCS-alone and scopolamine-plus-DCS conditions. For the DCS-alone condition, there was no main effect of drug and no dose $\times$ retention interval interaction. However, performance decreased systematically as a function of retention interval duration $[F(4,8)=8.45]$. In the scopolamine-plus-DCS condition, there were no effects of dose and no dose $\times$ retention interval interaction for the proportion correct measure. There was, however, a main effect of retention interval duration $[F(4,8)=8.54]$ whereby performance decreased systematically with increasing retention interval duration.

Figure 2 shows discriminability measures $(\log d)$ and fits of the exponential function for the DCS-alone condition. Consistent with Figure 1, no dose level of DCS improved performance above that of vehicle, and there was no dose $\times$ retention interval interaction. However, discriminability decreased as the retention interval lengthened $[F(4,8)=10.78]$.

In order to examine whether DCS alleviated the deficit generated by scopolamine in terms of the discriminability measure, the retention interval functions for each dose level of DCS combined with scopolamine were compared with the retention function for the vehicle control condition. This comparison is shown in Figure 3, where each panel includes the same functions for the vehicle control and scopolamine-plus-vehicle conditions (to show the deficit caused by scopolamine), along with the function for a particular dose of DCS combined with scopolamine.

Figure 3 shows that compared with the vehicle-control condition (circles), scopolamine significantly disrupted performance (squares), as indicated by the significant interaction between scopolamine-plus-vehicle versus vehicle control and retention interval duration $[F(4,8)=$
14.13]. At each delay for each bird, discriminability for the scopolamine condition was lower than in the vehiclecontrol condition. When DCS was administered in combination with scopolamine, there was a significant disruption in performance due to increasing retention interval duration $[F(4,8)=4.26]$, as well as a significant dose $X$ retention interval interaction $[F(12,24)=10.78]$, suggesting a delay-dependent change in performance as a function of dose. An important feature of the functions in Figure 3 is that discriminability at the 0.2 -sec retention interval was higher when DCS was combined with scopolamine than when scopolamine was administered with vehicle. This was the case for each dose level of DCS and for each bird, with the single exception of the $6-\mathrm{mg} / \mathrm{kg}$ dose for 1 bird. The most effective dose level at alleviating the scopolamine-induced deficit was $2 \mathrm{mg} / \mathrm{kg}$ of DCS $[F(4,8)=13.49]$.

Figure 4 summarizes the results of the present experiment in terms of the means of the parameter values for

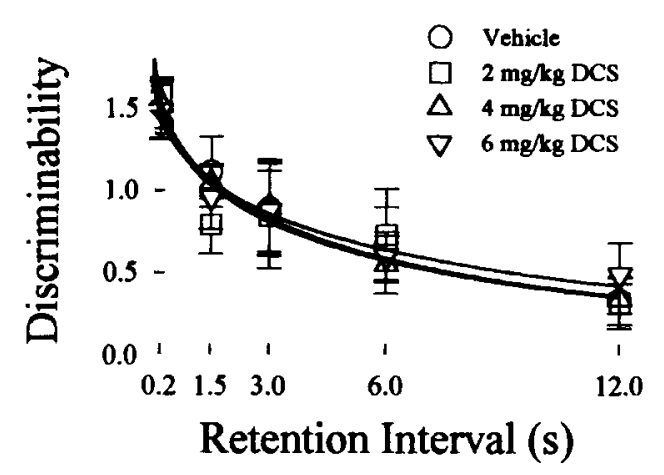

Figure 2. Group average discriminability $(\log d)$ as a function of retention interval for each dose level of D-cycloserine (DCS) alone. Vertical lines refer to standard errors of the mean. 

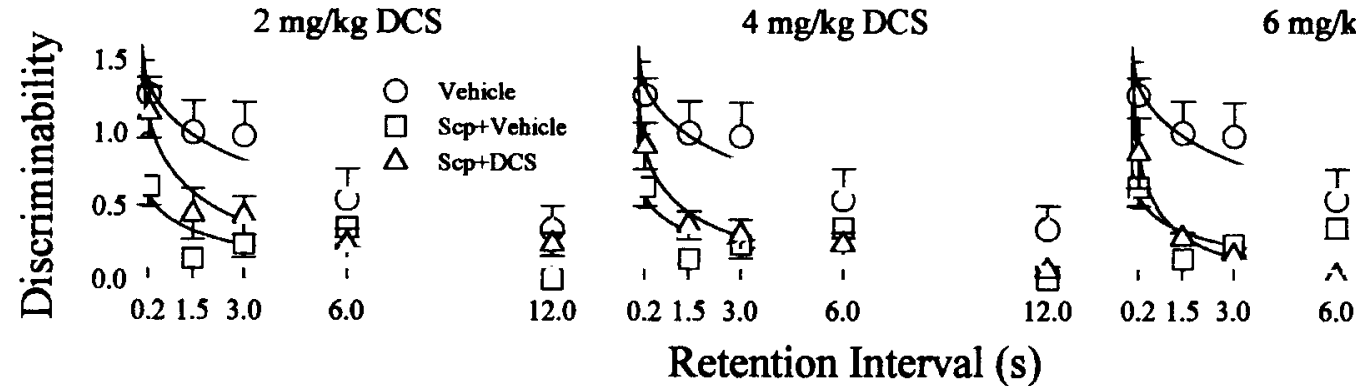

$\bigsqcup_{12.0}^{\nwarrow}$

Retention Interval (s)

Figure 3. Group average discriminability $(\log d)$ as a function of retention interval for each dose level of D-cycloserine (DCS) combined with scopolamine. Vertical lines refer to standard errors of the mean.

the exponential functions that best fit the data for individual birds. The intercept provides a measure of initial discriminability, and the slope parameter provides a measure of rate of forgetting. The top panels of Figure 4 show that neither initial discriminability nor rate of forgetting changed across the different dose levels of DCS when it was administered alone.

The bottom left panel of Figure 4 shows the marked disruption to initial discriminability when performance during scopolamine plus vehicle was compared with that of the vehicle control $[F(1,2)=74.43]$. This disruption was alleviated by administration of DCS in that the values for initial discriminability for each dose level of DCS combined with scopolamine were higher than for the scopolamine-plus-vehicle condition for each bird (with the exception of the $6-\mathrm{mg} / \mathrm{kg}$ dose for 1 bird). Alleviation of the scopolamine-induced disruption was statistically significant for the administration of $2 \mathrm{mg} / \mathrm{kg}$ of DCS $[F(1,2)=106.62]$. There was no consistent effect on rate of forgetting, either for the comparison between scopolamine-plus-vehicle and vehicle-control conditions or for the DCS-alone conditions.

\section{DISCUSSION}

DCS administered on its own did not significantly improve matching accuracy at any dose level tested. The failure of DCS to enhance performance in normal healthy rats has been reported before (Ruske, Harper, Colombo, \& White, 1995). It may be the case that the cognitively

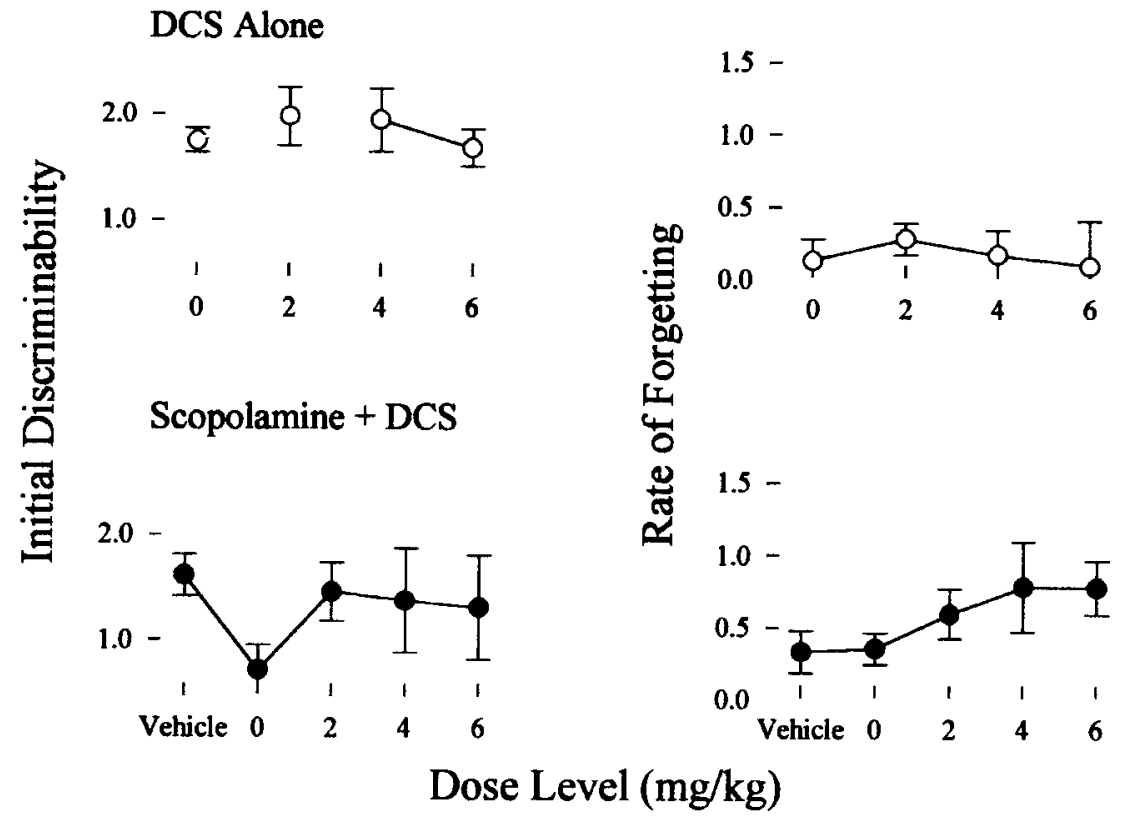

Figure 4. Group average initial discriminability and rate of forgetting parameters as a function of dose level for D-cycloserine (DCS) -alone and scopolamine-plus-DCS conditions. Vertical lines refer to standard errors of the mean. 
enhancing properties of DCS are restricted to situations where performance deficits have been experimentally induced, either by drugs or lesions.

Administration of scopolamine had a marked detrimental effect on matching accuracy. Consistent with previous studies with pigeons, rats, and primates (Kirk et al., 1988; White et al., 1996), this effect was manifest as a decrease in initial discriminability, without a reliable change in rate of forgetting. That is, discriminability at short retention intervals shows the greatest decrement with scopolamine administration, with the result that the $y$-intercept of the fitted exponential functions are overall lower. The overall deficit in memory performance is best characterized as involving attentional or encoding processes. An attentional deficit makes intuitive sense, even without the use of higher order analyses, given the consistent disruption in matching accuracy at the shortest retention interval duration of $0.2 \mathrm{sec}$. The cognitive deficit produced by scopolamine administration supports previous work showing attentional, rather than mnemonic deficits, following cholinergic system disruption (Bartus, 1978; Dunnett, 1985; Glick \& Jarvik, 1970; Kirk et al., 1988; Miyamoto et al., 1996; Penetar \& McDonough, 1983; Ruske et al., 1997; Safer \& Allen, 1971). Further, recent reviews of the role of the cholinergic system in learning and memory suggest that attentional rather than memorial processes are governed by cholinergic function (Blokland, 1996; Everitt \& Robbins, 1997).

The main finding of the present research was the ability of DCS to significantly attenuate the deficit induced by scopolamine. This is consistent with previous studies that have also shown amelioration of scopolamine-induced deficits following DCS administration (Jones et al., 1993; Matsuoka \& Aigner, 1996; Ohno \& Watanabe, 1996). In particular, when combined with scopolamine, DCS affected initial discriminability, consistent with the result that the scopolamine-induced deficit is manifest as an effect on initial discriminability. In other words, the same cognitive process can be assumed to be influenced by both a cholinergic antagonist and a glutamatergic agonist. Attenuation of cognitive performance in animals following glutamatergic administration has not been restricted to a scopolamine model of cholinergic system disruption, however. Glutamatergic agonists have also been effective at alleviating impaired performance induced by lesions to the hippocampus (Schuster \& Schmidt, 1992) and entorhinal cortex (Myhrer, 1993; Myhrer \& Paulsen, 1992).

The findings from the present study suggest that NMDA receptor agonists may be useful for alleviating the cognitive deficits arising from cholinergic system disruption. Although it is impossible to ascertain the mechanism through which DCS produced its enhancing effect, a plausible explanation may be its action at the NMDA receptor site, and therefore its ability to enhance or prolong the induction of LTP. For example, Barnes (1995) referred to LTP as the "artificially induced phenomenon that reflects the activation of processes that are actually used for the encoding of memory" (p. 751). This is supported by studies that have found encoding rather than rate-of-forgetting changes in conditional discrimination tasks following the administration of NMDA receptor antagonists. For example, Tan, Kirk, Abraham, and McNaughton (1989) found that two NMDA antagonists, CPP (a competitive NMDA antagonist) and MK-801 (a noncompetitive NMDA antagonist), disrupted the ability of rats to perform accurately in an auditory delayed conditional discrimination procedure. They reported reduced initial discriminability values, suggesting that encoding processes were disrupted by drug administration. They did not detect any change in the rate-of-forgetting parameter. Similarly, in a delayed matching-to-sample procedure using rats, Ruske et al. (1995) reported that MK-801 impaired matching accuracy and that the deficit was due to a reduction in initial discriminability.

Some evidence also suggests that one action of $\mathrm{ACh}$ is to facilitate LTP induction (Burgard \& Sarvey, 1990). There are suggestions that the ability of glutamatergic agonists to reverse cholinergic system dysfunction might be due to a postsynaptic interaction between the cholinergic and glutamatergic systems (Ohno \& Watanabe, 1996). This is possible given that Markram and Segal (1990) demonstrated that acetylcholine administration produces an increase in NMDA receptor-mediated responses by its ability to facilitate excitatory postsynaptic potentials. This suggests that one of the functions of cholinergic activity is to modulate glutamatergic transmission. Future research investigating the mechanisms through which the cholinergic and glutamatergic systems interact would be needed to determine their exact role in cognitive function.

\section{REFERENCES}

ABraham, W. C. (1988). Long-term potentiation as a possible associative memory mechanism in the brain. New Zealand Journal of Psychology, 17, 49-57.

Abraham, W. C., \& Mason, S. E. (1988). Effects of the NMDA receptor antagonists CPP and MK-801 on hippocampal field potentials in long-term potentiation in urethane anaesthetized rats. Brain Research, 462, 40-46.

Aigner, T. G. (1995). Pharmacology of memory: Cholinergicglutamatergic interactions. Current Opinions in Neurobiology, 5 , 155-160.

BARNES, C. A. (1995). Involvement of LTP in memory: Are we "searching under the street light?" Neuron, 15, 751-754.

BARTUS, R. T. (1978). Evidence for a direct cholinergic involvement in the scopolamine-induced amnesia in monkeys: Effects of concurrent administration of physostigmine and methylphenidate with scopolamine. Pharmacology. Biochemistry \& Behavior, 9, 833-836.

Baxter, M. G., Lanthorn, T. H., Frick, K. M., Golski, S., Wan, R., \& Olton, D. S. (1994). D-Cycloserine, a novel cognitive enhancer, improves spatial memory in aged rats. Neurobiology of Aging, 15, 207-213.

Blitzer, R. D., Gil, O., \& Landau, E. M. (1990). Long-term potentiation in rat hippocampus is inhibited by low concentrations of ethanol. Brain Research, 537, 203-208.

BLOKLAND, A. (1996). Acetylcholine: A neurotransmitter for learning and memory? Brain Research Reviews, 21, 285-300. 
Burgard, E. C., \& SARvey, J. M. (1990). Muscarinic receptor activation facilitates the induction of long-term potentiation (LTP) in the rat dentate gyrus. Neuroscience Letters, 116, 34-39.

Collingridge, G. L., \& Bliss, T. V. P. (1987). NMDA receptorsTheir role in long-term potentiation. Trends in Neurosciences, 10 , 288-293.

Davis, S., Butcher, S. P., \& Morris, R. G. M. (1992). The NMDA receptor antagonist D-2-amino-5-phosphonopentanoate (D-APV) impairs spatial learning and LTP in vivo at intracerebral concentrations comparable to those that block LTP in vitro. Journal of Neuroscience, 12, $21-34$.

DunNeTt, S. B. (1985). Comparative effects of cholinergic drugs and lesions of nucleus basalis or fimbria-fornix on delayed matching in rats. Psychopharmacology, 87, 357-363.

EveritT, B. J., \& RobBINs, T. W. (1997). Central cholinergic systems and cognition. Annual Review of Psychology, 48, 649-684.

FloOD, J. F., BAKER, M. L., \& DAVIS, J. L. (1990). Modulation of memory processing by glutamic acid receptor agonists and antagonists. Brain Research, 521, 197-202.

GliCK, S. D., \& JARVIK, M. E. (1970). Differential effects of amphetamine and scopolamine upon matching performance of monkeys with lateral frontal lesions. Journal of Comparative Physiological Psychology, 73, 307-313.

Handelmann, G. E., Nevins, M. E., Mueller, L. L., Arnolde, S. M., \& CORDI, A. A. (1989). Milacemide, a glycine prodrug, enhances performance of learning tasks in normal and amnestic rodents. Pharmacology, Biochemistry \& Behavior, 34, 823-828.

Hautus, M. J. (1995). Corrections for extreme proportions and their biasing effects on estimated values of $d^{\prime}$. Behavioral Research Methods, Instruments, \& Computers, 27, 46-51.

Ito, T., Miura, Y., \& KadokaWa, T. (1988). Physostigmine induces in rats a phenomenon resembling long-term potentiation. European Journal of Pharmacology, 156, 351-359.

IVERSEN, S. D. (1997). Behavioural evaluation of cholinergic drugs. Life Sciences, 60, 1145-1152.

JONES, R. W., WESNES, K. A., \& KIRBY, J. (1993). Effects of NMDA modulation in scopolamine dementia. In P. Tallal et al. (Eds.), Temporal information processing in the nervous system: Special reference to dyslexia and dysphasia (Annals of the New York Academy of Sciences, Vol. 682, 39-46). New York: New York Academy of Sciences.

Kirk, R. C., White, K. G., \& McNaughton, N. (1988). Low dose scopolamine affects discriminability but not rate of forgetting in delayed conditional discrimination. Psychopharmacology, 96, 541-546.

Levkovitz, Y., \& Segal, M. (1994). Acetylcholine mediates the effects of fenfluramine on dentate granule cell excitability in the rat. European Journal of Pharmacology, 264, 279-284.

Markram, H., \& Segal, M. (1990). Long-lasting facilitation of excitatory postsynaptic potentials in the rat hippocampus by acetylcholine. Journal of Physiology, 427, 381-393.

Matsuoka, N., \& Aigner, T. G. (1996). D-Cycloserine, a partial agonist at the glycine site coupled to $N$-methyl-D-aspartate receptors, improves visual recognition memory in rhesus monkeys. Journal of Pharmacology \& Experimental Therapeutics, 278, 891-897.

MiYamoto, M., TaKahashi, H., Kato, K., Hirai, K., Ishihara, Y., \& GoTo, G. (1996). Effects of 3-[1-(phenylmethyl)-4-(piperidinyl)]-1 (2,3,4,5-tetrahydro-1 H-1-benzazepin-8-yl)-1-propanone fumarate (TAK-147), a novel acetylcholinesterase inhibitor, on impaired learning and memory in animal models. Journal of Pharmacology \& Experimental Therapeutics, 277, 1292-1304.

Monahan, J. B., Handelmann, G. E., Hood, W. F., \& Cordi, A. A. (1989). D-Cycloserine, a positive modulator of the $N$-methyl-D- aspartate receptor, enhances performance of learning tasks in rats. Pharmacology, Biochemistry \& Behavior, 34, 649-653.

MorRIS, R. G. M. (1989). Synaptic plasticity and learning: Selective impairment of learning in rats and blockade of long-term potentiation in vivo by the $\mathrm{N}$-methyl-D-aspartate receptor antagonist AP5. Journal of Neuroscience, $9,3040-3057$.

MyHrer, T. (1993). Animal models of Alzheimer's disease-Glutamatergic denervation as an alternative approach to cholinergic denervation. Neuroscience \& Biobehavioral Reviews, 17, 195-202.

Myhrer, T., \& Paulsen, R. E. (1992). Memory dysfunction following disruption of glutamatergic systems in the temporal region of the rat-Effects of agonistic amino acids. Brain Research, 599, 345-352.

Ohno, M., \& Watanabe, S. (1996). D-Cycloserine, a glycine site agonist, reverses working memory failure by hippocampal muscarinic receptor blockade in rats. European Journal of Pharmacology, 318, 267-271.

Penetar, D. M., \& McDonough, J. H. (1983). Effects of cholinergic drugs on delayed matching-to-sample performance of rhesus monkeys. Pharmacology, Biochemistry \& Behavior, 19, 963-967.

Ruske, A. C., Fisher, A., \& White, K. G. (1997). Attenuation of scopolamine-induced deficits in delayed-matching performance by a new muscarinic agonist. Psychobiology, 25, 313-320.

Ruske, A. C., Harper, D. N., Colombo, M. W., \& White, K. G. (1995). NMDA receptor action and spatial memory: The effects of MK-801 and D-cycloserine on rate of forgetting and initial discriminability. Psychobiology, 23, 277-283.

SAFER, D. J., \& ALLEN, R. P. (1971). The central effects of scopolamine in man. Biological Psychiatry, 3, 347-355.

SCHUSTER, G. M., \& SCHMIDT, W. J. (1992). D-Cycloserine reverses the working memory impairment of hippocampal-lesioned rats in a spatial learning task. European Journal of Pharmacology, 224, 97-98.

Segal, M., \& Auerbach, J. M. (1997). Muscarinic receptors involved in hippocampal plasticity. Life Sciences, 60, 1085-1091.

Tan, S., Kirk, R. C., Abraham, W. C., \& McNaughton, N. (1989). Effects of the NMDA antagonists CPP and MK-801 on delayed conditional discrimination. Psychopharmacology, 98, 556-560.

WATSON, J. E., \& BLAMPIED, N. M. (1989). Quantification of the effects of chlorpromazine on performance under delayed matching to sample in pigeons. Journal of the Experimental Analysis of Behavior, $\mathbf{5 1}$, 317-328.

WHITE, K. G. (1985). Characteristics of forgetting functions. Journal of the Experimental Analysis of Behavior, 44, 15-34.

WhITE, K. G. (1991). Psychophysics of direct remembering. In M. L. Commons, J. A. Nevin, \& M. C. Davison (Eds.), Signal detection: Mechanisms (pp. 221-237). Hillsdale, NJ: Erlbaum.

WhITE, K. G., \& HARPER, D. N. (1996). Quantitative reanalysis of lesion effects on rate of forgetting in macaques. Behavioural Brain Research, 74, 223-227.

White, K. G., Harper, D. N., \& Watson, J. E. (1994). Effects of chronic phenobarbital administration on forgetting functions in pigeons. Pharmacology, Biochemistry \& Behavior, 49, 427-431.

White, K. G., Ruske, A. C., \& Colombo, M. (1996). Memory procedures, performance, and processes in pigeons. Cognitive Brain Research, 3, 309-317.

Wong, E. H. F., Kemp, J. A., Priestley, T., Knight, A. R., Woodruff, G. N., \& IVERSEN, L. L. (1986). The anticonvulsant MK-801 is a potent $N$-methyl-D-aspartate antagonist. Proceedings of the National Academy of Sciences, 83, 7104-7108.

(Manuscript received July 28, 1998; revision accepted for publication March 29, 1999.) 